\title{
Meaningful Thickness Detection on Polygonal Curve
}

\author{
Bertrand Kerautret $^{1,2}$, Jacques-Olivier Lachaud ${ }^{2}$ and Mouhammad Said ${ }^{2}$ \\ ${ }^{1}$ LORIA (UMR CNRS 7503) Nancy University, France \\ ${ }^{2}$ LAMA (UMR CNRS 5127), University of Savoie, France \\ ${ }^{3}$ LIRIS (UMR CNRS 5205), University of Lyon 2, France \\ kerautre@loria.fr,Jacques-Olivier.Lachaud@univ-savoie.fr, Mouhammad.Said@univ-lyon2.fr
}

Keywords: shape analysis, noise detection, meaningful scale, contour representation

\begin{abstract}
The notion of meaningful scale was recently introduced to detect the amount of noise present along a digital contour. It relies on the asymptotic properties of the maximal digital straight segment primitive. Even though very useful, the method is restricted to digital contour data and is not able to process other types of geometric data like disconnected set of points. In this work, we propose a solution to overcome this limitation. It exploits another primitive called the Blurred Segment which controls the straight segment recognition precision of disconnected sets of points. The resulting noise detection provides precise results and is also simpler to implement. A first application of contour smoothing demonstrates the efficiency of the proposed method. The algorithms can also be tested online.
\end{abstract}

\section{Introduction}

Detecting if a contour is sampled at a meaningful scale and estimate what are the correct scales to analyse it (if they exist) is an important issue in shape analysis. For instance, it makes easier the automated parameterization in geometric shape analysis, contour representation or pattern recognition. In general, the noise is taken into account by a supervised parameter chosen according to the input data quality. The choice of the parameter is largely influential on the quality of the process. For instance in a deformable boundary segmentation technique, the smoothing parameter has a great impact on the result.

Following a principle of perception from the Gestalt theory, Desolneux et al. propose to detect the meaningful edges of a grey level image by using false alarm probability defined on the iso contours of the image (Desolneux et al., 2001). This detection is based on the image gradient and is not directly defined for discrete contour representations. Along the same lines, Cao introduces the notion of meaningful good continuation (Cao, 2003) which relies on geometric properties of the given curve. The false alarm probability was simply approximated by a curvature estimation of the input curve. Other applications of Gestalt theory can be found in a recent article (Desolneux, 2011).

The concept of meaningful scale along digital

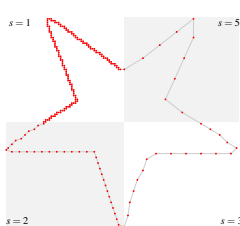

(a)

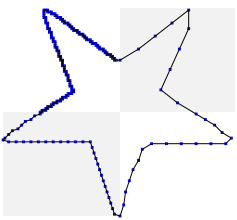

(e)

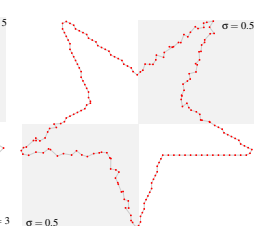

(b) $s=3$

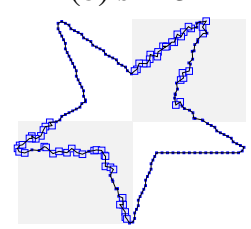

(f)

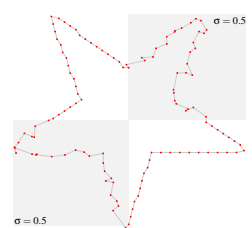

(c) $s=5$

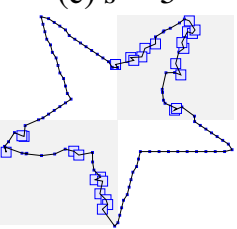

(g)
Figure 1: Noise detection on polygonal curves defined both with different sampling rate $(s)$, image (a) and with several gaussian noise intensity $(\sigma)$, images $(b, c)$. The resulting noise detection of polygons (a-c) is given on (e-f) where the detected noise level is represented by boxes.

contour (i.e. boundary of a digital region) has been introduced in a recent work to detect automatically if the current scale is locally significant or not (Kerautret and Lachaud, 2009b). It relies on the study of asymptotic properties of maximal digital straight segment which is a classic tool used to extract geometric parameter on a discrete contour (Lachaud et al., 2005). More precisely the length of discrete maximal segment is analyzed on the contour represented at different scales to determine locally if a point is mean- 
ingful or not. In opposition with the work of $\mathrm{Cao}$, the method can also detect what is the local best scale to analyze the considered shape (if it exists).

The limitation of the meaningful scale method lies mainly in the fact that the analysis is only possible on a sequence of simple 4 or 8 -connected points and cannot be applied on a general polygon such the ones illustrated on Fig. 1. This restriction is due to the intrinsic properties of the discrete primitive. In this work, we introduce the new notion of meaningful thickness by using a less restrictive primitive (the $\alpha$-thick Blurred Segment) that is described in the next section.

\section{Maximal Digital and Blurred Straight Segment}

Introduced in the 1970's, the digital straightness has been an active topic studied through many years, see for instance (Rosenfeld, 1974; Dorst and Smeulders, 1984; Bruckstein, 1991), and (Klette and Rosenfeld, 2004) for a recent review. Its potential applications are numerous from the definition of geometric estimators like tangent, curvature (Kerautret and Lachaud, 2009a) to for instance polygonal contour representation (Bhowmick and Bhattacharya, 2007). Although there are different definitions, we recall the classic standard digital straight line (DSL) primitive used in the concept of the meaningful scale detection.

A standard Digital Straight Line (DSL) is some set $\left\{(x, y) \in \mathbb{Z}^{2}, \mu \leq a x-b y<\mu+|a|+|b|\right\}$, where $(a, b, \mu)$ are also integers and $\operatorname{gcd}(a, b)=1$. It is well known that a DSL is a 4-connected simple path in the digital plane. A digital straight segment (DSS) is a 4-connected piece of DSL. The interpixel contour of a simple digital shape is a 4-connected closed path without self-intersections. Given such a 4-connected path $C$, a maximal segment $M$ is a subset of $C$ that is a DSS and which is no more a DSS when adding any other point of $C \backslash M$.

A recognition process of a DSS is illustrated on Fig. 2 (a) where a maximal segment is recognized by adding step by step the sequence of points: $P_{1}, Q_{1}$, $P_{2}, Q_{2}, P_{3}, Q_{3}, P_{4}, Q_{4}, P_{5}, Q_{5}, Q_{6}, Q_{7}, Q_{8}, Q_{9}$. After adding the point $P_{5}$ the DSS can only be extended on the back (points $Q_{i}$ ) since the point $P_{6}$ does not belong to the segment. The discrete length of this DDS is defined as the number of step and is denoted as $L_{j}$ where $j$ is DSS number covering an initial point $\left(L_{0}=\right.$ 14 in the example of Fig. 2).
Blurred segments were introduced to address noisy data (Debled-Rennesson et al., 2006). We use the following definition (Faure et al., 2009): a set of points

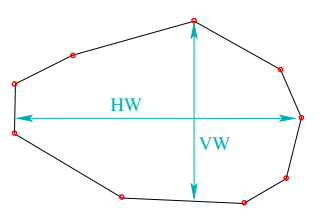
is an $\alpha$-thick Blurred Segment if and only if its convex hull has an isothetic thickness less than a given real number $\alpha$. The isothetic thickness of a convex hull is the smallest value between its vertical and its horizontal width (denoted respectively as HW and VW on the upper floating figure). In the same way as previously a maximal $\alpha$-thick Blurred Segment can be defined as a segment which can not be extended to the front or to the back.

An illustration is given on Fig. 2 (b). An $\alpha$-thick Blurred Segment with $\alpha=1$ is recognized from the point $A$ by adding alternatively the points $P_{1}, Q_{1}, P_{2}$, $Q_{2}$ and $P_{3}$ (denoted as 1-thick $B S_{1}$ ). Neither the points $P_{4}$ nor $Q_{3}$ can be added to the maximal 1-thick $B S_{1}$ since the resulting isothetic thickness will be greater than $\alpha=1$. Another maximal segment 1-thick $B S_{0}$ covering the point $A$ is illustrated in light color on Fig. 2 (b). For each segment 1-thick $B S_{i}$, its length $\mathcal{L}_{i}^{1}$ is illustrated in light gray and constitutes an essential property which will be exploited in the definition of meaningful thickness introduced in the next section.

\section{Meaningful Thickness Detection with Maximal Blurred Segment}

Before introducing the new concept of Meaningful Thickness we recall briefly the main idea of the meaningful scale (Kerautret and Lachaud, 2009b) detection and show the main inconvenient.

\subsection{Asymptotic property of maximal segments}

The meaningful scale detection relies on the analysis of asymptotic property of maximal straight segments. This property is the discrete length $\left(L_{j}^{h}\right)$ of a maximal segment belonging to a contour point given at a digitization grid size $h$. In the following, we will denote by $\operatorname{Dig}_{h}(S)$ the Gauss digitization process $\left(\operatorname{Dig}_{h}(S)=X \cap h \mathbb{Z} \times h \mathbb{Z}\right)$. From different analysis shown in (Lachaud, 2006; Kerautret and Lachaud, 2009b), several properties can be summed up as follows:

Property 1. Let $S$ be a simply connected shape in $\mathbb{R}^{2}$ with a piecewise $C^{3}$ boundary. Let $P$ be a point of the 


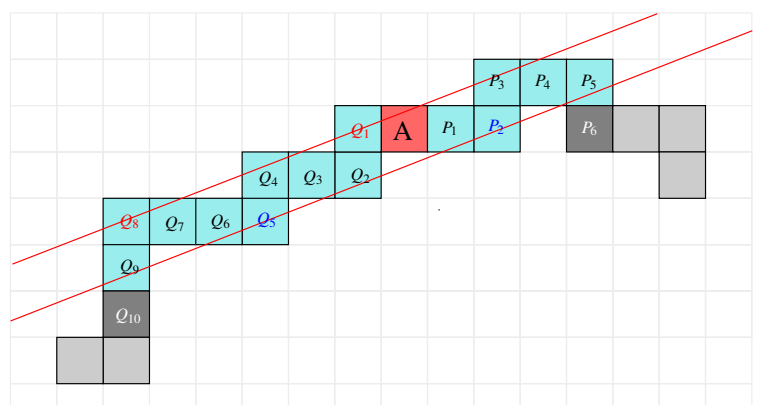

(a)

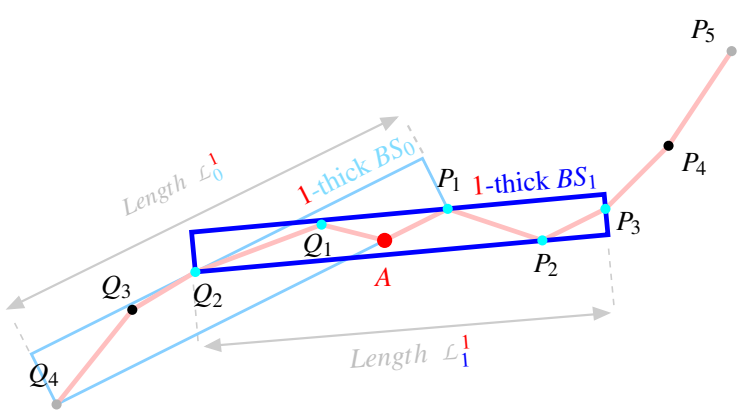

(b)

Figure 2: Illustration of (a) a maximal DSS of characteristics $(a, b, \mu)=(2,5,0)$, and (b) two $\alpha$-thick Blurred Segments with $\alpha=1$ (denoted as 1-thick $B S_{i}$ with $i=0,1$ ).

boundary $\partial S$ of $S$. Consider now an open connected neighborhood $U$ of $P$ on $\partial S$. Let $\left(L_{j}^{h}\right)$ be the digital lengths of the maximal segments along the boundary of $\operatorname{Dig}_{h}(S)$ and which cover $P$. Then, the asymptotic behaviour of the digital lengths follows these bounds: if $U$ is strictly convex or concave, then

$$
\Omega\left(1 / h^{1 / 3}\right) \leq L_{j}^{h} \leq O\left(1 / h^{1 / 2}\right)
$$

if $U$ has null curvature, then

$$
\Omega(1 / h) \leq L_{j}^{h} \leq O(1 / h)
$$

The strategy to exploit this property was to transform the initial discrete contour with several grid sizes $h$ while keeping the point associations and checking the discrete contour consistency. The resulting analysis shows precise and fine noise detection but is however not general for the analysis of other type of non discrete contours.

A natural idea to generalize the analysis to polygonal contour is to consider the primitive of the $\alpha$ thick Blurred Segment described in the previous section which allows to deal with non integer points and not necessary connected. The primitive presents another advantage with its thickness parameter $\alpha$ that can be used as a scale parameter.

\subsection{Thickness asymptotic properties of Blurred Segments}

To define the notion of Meaningful Thickness with the $\alpha$-thick Blurred Segment, we need first to focus on the asymptotic properties of the blurred segments in the multi-thickness decomposition of a given contour. The Euclidean length $\mathcal{L}$ will replace the digital length $L$ used in the previous Property $1 . \mathcal{L}$ is defined as the length of the bounding box obtained from the $\alpha$-thick Blurred Segment convex hull. Fig. 2 (b) illustrates such a bounding box with the length of two 1-thick Blurred Segments covering the point A (1thick $B S_{0}$ and 1-thick $\left.B S_{1}\right)$. Their bounding boxes are given respectively by the points $P_{1}, Q_{1}, Q_{2}, Q_{3}, Q_{4}$ and $Q_{2}, P_{2}, P_{3}, P_{1}, Q_{1}$

When the Euclidean lengths of blurred segments around a point of a polygonal contour is computed, we observe an increasing sequence of lengths for the increasing sequence of real thicknesses $t_{i}=i k \sqrt{2}$ where $k$ is the mean distance between consecutive polygon vertices. When plotted in logscale, its slope is related to the localization of the point in a flat or curved zone. More precisely, letting $\left(\mathcal{L}_{j}^{t_{i}}\right)_{j=1, \ldots, l_{i}}$ be the Euclidean lengths of the blurred segments along the digital contour and covering a point, we have observed experimentally the following behavior:

Property 2. (Multi-thickness). The plots of the lengths $\mathcal{L}_{j}^{t_{i}} / t_{i}$ in log-scale are approximately affine with negative slopes as specified besides:

\begin{tabular}{|c|c|c|}
\cline { 2 - 3 } \multicolumn{1}{c|}{} & \multicolumn{2}{c|}{ expected slope } \\
\hline plot & curved part & flat part \\
\hline$\left(\log \left(t_{i}\right), \log \left(\max _{j} \mathcal{L}_{j}^{t_{i}} / t_{i}\right)\right)$ & $\approx-\frac{1}{2}$ & $\approx-1$ \\
\hline$\left(\log \left(t_{i}\right), \log \left(\min _{j} \mathcal{L}_{j}^{t_{i}} / t_{i}\right)\right)$ & $\approx-\frac{1}{3}$ & $\approx-1$ \\
\hline
\end{tabular}

Fig. 4 and Fig. 3 illustrates such a behaviour on an ellipse shape represented by a disconnected set of points with some missing parts. The set of $\alpha$-thick Blurred Segments covering a specific point $P\left(l_{i}\right.$ segments cover $P$ ) is represented on Fig. 4 with four different thicknesses $(t=\sqrt{2}, 2 \sqrt{2}, 3 \sqrt{2}, 4 \sqrt{2})$. For each thickness $t_{i}$, the lengths $\left(L_{j}^{t_{i}}\right)_{j=1, \ldots, l_{i}}$ are represented on the plot of Fig. 4. On this simple example we can check that the segment length verifies the previous Property 2 with their $\min$ and $\max$ values near the slope $-\frac{1}{2}$ and $-\frac{1}{3}$ which correspond to the hypothesis of a curved contour part. Other measures on different shapes are given in (Said, 2010).

As in the multi-scale computation (Kerautret and Lachaud, 2009b), the multi-thickness results allows 


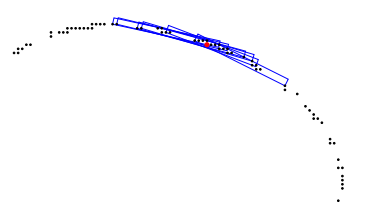

(a) $t_{1}=\sqrt{2}$

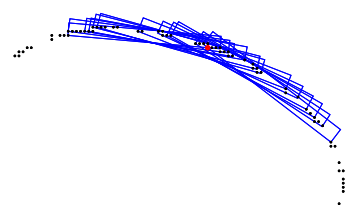

(b) $t_{2}=2 \sqrt{2}$

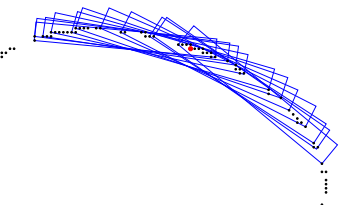

(c) $t_{3}=3 \sqrt{2}$

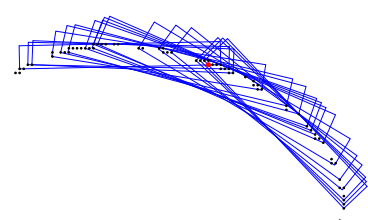

(c) $t_{4}=4 \sqrt{2}$

Figure 3: Covering a point of the initial contour by the blurred segments obtained with different thicknesses $\left(t_{i}\right)$. For each thickness the blurred segments covering the considered point (drawn in red) are drawn with blue boxes.

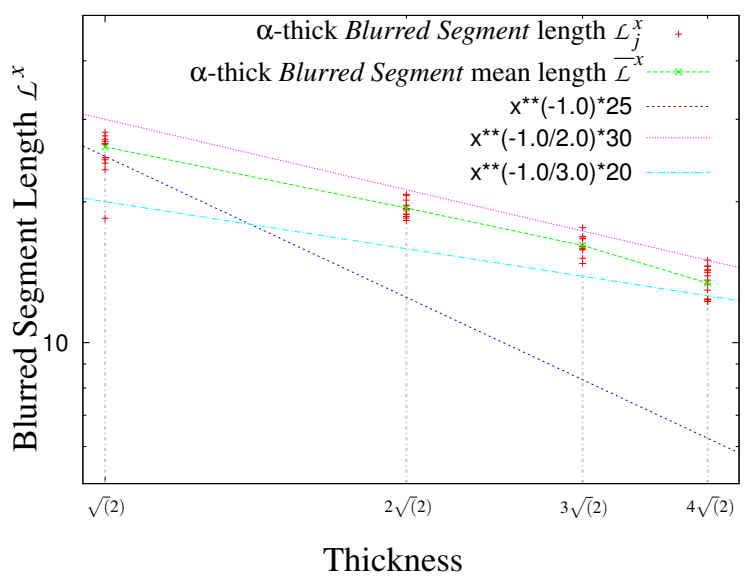

Figure 4: Illustration of the Lengths $\mathcal{L}_{j}^{x}$ from the set of $\alpha$-thick Blurred Segments given from illustration of Fig. 3. The lines of slope $-1,-\frac{1}{2}$ and $-\frac{1}{3}$ are also given to illustrate the reference constraint of Property 2.

us to distinguish between curved and flat parts of an object boundary. This approach is not valid on points that are (1) a transition between a curved and a flat part, (2) corner points. Finally, this technique assumes smooth objects with perfect digitization: if the digital contour has been damaged by noise or digitization artefact, these characterizations do not hold.

Although the two last remarks seem problematic for analyzing shapes, we will use them to detect $l o$ cally the amount of noise and to extract the local meaningful thickness.

\subsection{Local geometric evaluation with multi-thickness criterion}

We analyze now the local geometry of some point $P$ on a polygonal curve $C$ having a mean distance between vertices equals to $k$. For various values of thickness $t_{i}=i k \sqrt{2}, i=1 . . n$, we compute the Euclidean lengths $L_{j}^{t_{i}}$ of the blurred segments of $C$. For a given thickness $i k \sqrt{2}$, the average length of all blurred segments covering a point $P$ is denoted as $\overline{\mathcal{L}}^{t_{i}}=\frac{1}{l_{i}} \sum_{j} \mathcal{L}_{j}^{t_{i}}$, where $l_{i}$ represents the number of blurred segments containing $P$. Fig. 3 illustrates the blurred segments covering a point, obtained on the contour with several values of thickness $t_{i}$.

We define the multi-thickness profile $\mathcal{P}_{n}(P)$ of a point $P$ as the graph $\left(\log \left(t_{i}\right), \log \left(\bar{L}^{t} t_{i} / t_{i}\right)\right)_{i=1, \ldots, n}$. We also define the ideal multi-thickness criterion $\mu_{n}(P)$ of a point $P$ on the boundary of a digital object as the slope coefficient of the simple linear regression of $\mathcal{P}_{n}(P)$. Property 2 indicates that $\mu_{n}(P)$ should be around -1 if $P$ is in flat zone, whereas it should be within $[-1 / 2,-1 / 3]$ if $P$ is in a strictly convex or concave zone.

Detecting noise and local meaningful thickness. The multi-thickness profile can be used to detect noisy polygonal curves. We show on Fig. 5 (b) the multi-thickness profile of a point $P_{A}$ located on a perfectly digitized curved zone and the multi-thickness profiles of the points $P_{B}, P_{C}$ and $P_{D}$ located in noisy zones (image (a)). On the first profile, the decreasing affine relation is immediately visible. On the latter profiles, it is increasing at fine resolution and then falls back on a decreasing affine profile after a given thickness. We apply also the multi-thickness profiles on the polygonal curve with flat and curved areas (Fig. 5 (c,d)). The difference between them is the slope of the affine relation of the profiles (slope near $-\frac{1}{2}$ for the plots of points $P_{C}, P_{D}$ and $P_{E}$ and near -1 for the plots of points $P_{A}$ and $P_{B}$ ).

We then introduce a noise threshold $T_{m}$ which discriminates between a curved zone and a noisy zone. This threshold should be somewhere between ]$-\frac{1}{3}, 0[$. However after several experiments on noisy shapes it appears that the use of the upper threshold value $T_{m}=0$ gives best results both on curved or flat noisy parts.

A meaningful thickness of a multi-thickness profile $\left(X_{i}, Y_{i}\right)_{1 \leq i \leq n}$ is then a pair $\left(i_{1}, i_{2}\right), 1 \leq i_{1}<i_{2} \leq n$, such that for all $i, i_{1} \leq i<i_{2}, \frac{Y_{i+1}-Y_{i}}{X_{i+1}-X_{i}} \leq T_{m}$, and the property is not true for $i_{1}-1$ and $i_{2}$. The first meaningful thickness $\left(i_{1}\right)$ of a point $P$ can be considered as a noise level and is denoted as $t_{\tau}(P)=i_{1}$. Note that this noise level definition is the independant to 


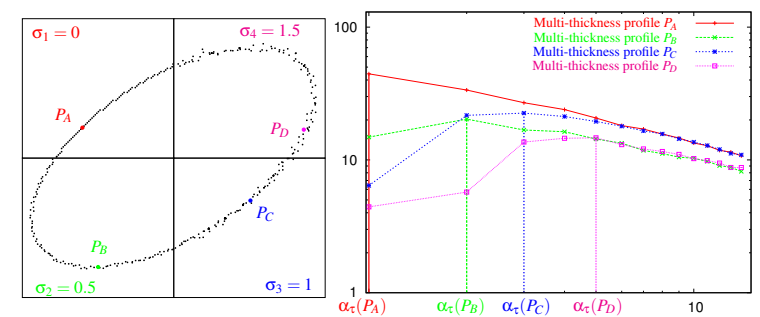

(a)

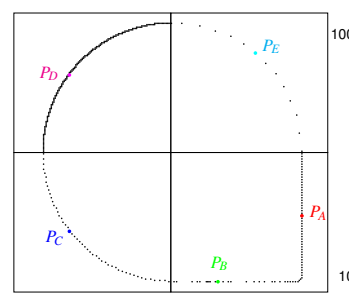

(c) (b)

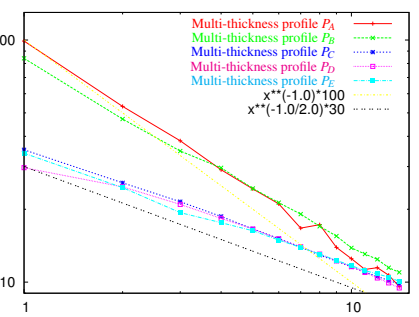

(d)

Figure 5: Multi-thickness profiles (b,d) obtained on two sampled contours $(\mathrm{a}, \mathrm{c})$. The curve (a) was obtained by adding gaussian noise with std deviation $\sigma$.

the second value $i_{2}$.

From the example of Fig. 5 (a,b), the point $A$ located on a smooth contour part, has a meaningful thickness equals to $(1 \sqrt{2}, 14 \sqrt{2})$ with $t_{\tau}(A)=1 \sqrt{2}$. For the noisy contour parts, the points $P_{B}, P_{C}$ and $P_{D}$ have respectively a meaningful thickness equals to $(2 \sqrt{2}, 14 \sqrt{2}),(3 \sqrt{2}, 14 \sqrt{2})$ and $(5 \sqrt{2}, 14 \sqrt{2})$. This example show that the meaningful thickness is well identified and is related on the noise intensity. Moreover the other example of Fig. 5 (c,d) demonstrates that the meaningful thickness detection is not degraded by changes of the sampling rate. This experiment will be also confirmed in the next section.

To improve the notion ideal multi-thickness criteria on noisy data, we adapt it with the use the previous meaningful thickness. Then, if $\left(i_{1}, i_{2}\right)$ is a meaningful thickness of some profile $P_{n}(P)$, the $\left(i_{1}, i_{2}\right)$-multithickness criterion $\mu_{i_{1}, i_{2}}(P)$ of point $P$ is then the slope coefficient of the simple linear regression of $P_{n}(P)$ restricted to its samples from $i_{1}$ to $i_{2}$. This definition will be used in experiments of the following section.

\section{Experiment and Comparison}

Before applying comparisons of the meaningful thickness detection with the meaningful scale approach, it is important to measure the influence of the parameter used in the method. The first parameter is the maximal thickness $\left(t_{\max }\right)$ used to create the meaningful thickness profile and the second one is the

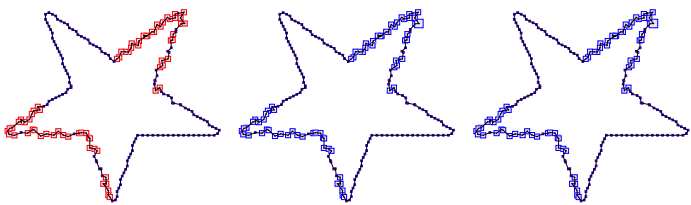

(a) $t_{\max }=5 \sqrt{2}$ (b) $t_{\max }=10 \sqrt{2}(c) t_{\max }=15 \sqrt{2}$
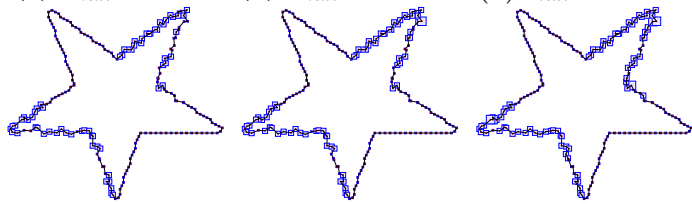

(d) $T_{m}=0.2$

(e) $T_{m}=0.0$

(f) $T_{m}=-0.2$

Figure 6: Evaluation of the independence of the meaningful thickness detection from the different parameters. The size of the blue boxes represents for each point the obtained meaningful scale or thickness $\alpha_{\tau}$. The first row presents the evaluation by varying the maximal thickness used to define the multi-thickness profile $\left(t_{\max }\right)$. The red color indicates present on image (a) indicates that there exists no meaningful thickness less than $t_{\max }$. The second row shows the stability by the change of the noise threshold parameter $T_{m}$.

minimal slope to consider a point as noise (parameter $\left.T_{m}\right)$

The first experiment of Fig. 6 (a-c) shows that the parameter $t_{\max }$ does not change the quality of the detection. The images $(b, c)$ show quite similar noise levels. For the first experiment (a) the pixels drawn in red show that no meaningful thickness was found since the maximal value $t_{\max }=5 \sqrt{2}$ was too small and the meaningful thickness is in fact greater than $t_{\max }$. The stability for the other parameter $T_{m}$ was also experimented on Fig. 6 (d-f). The default value of $T_{m}$ set to 0 was experimented as giving best results but we can see that a large change of this parameter does not really change the noise detection quality. Other experiments ${ }^{1}$ confirm that the proposed method can be considered as parameter free. Note that for all other experiments these parameters were set to $t_{\max }=15$ and $T_{m}=0$ (and also in the online demonstration).

\subsection{Experiment of meaningful thickness detection}

Comparison with the meaningful scale. To evaluate the quality of the meaningful thickness detection we perform some comparisons with the meaningful scale detection. Fig. 7 presents results on a digital shape where noise was added manually to the initial curve. The detection accuracy appears as precise as the meaningful scales if we except some corners of

\footnotetext{
${ }^{1}$ Other experiments can be done online (Kerautret et al., 2011)
} 


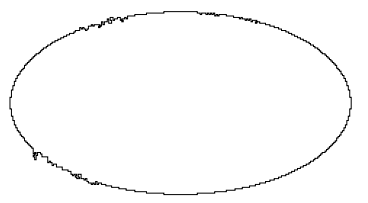

(a) 828 points

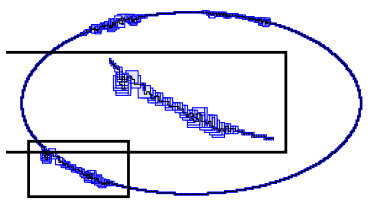

(b) Meaningful scale

Lachaud, 2009b)

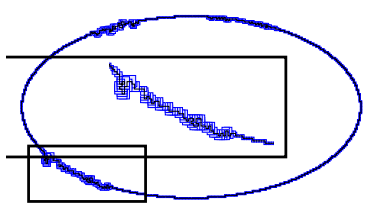

(c) Meaningful thickness.
(Kerautret

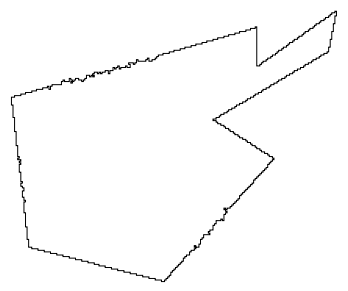

(d) 966 points

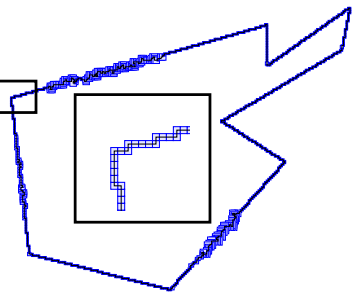

(e) Meaningful scale

(Kerautret

Lachaud, 2009b)

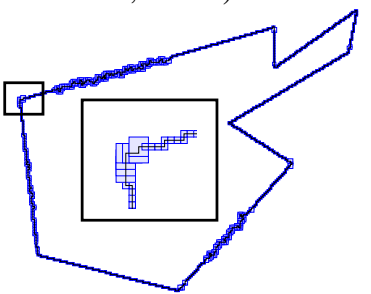

(f) Meaningful thickness.
Figure 7: Comparison between meaningful scale (Kerautret and Lachaud, 2009b) (second line) and meaningful thickness (third line). The size of the blue boxes represents for each pixel the obtained meaningful scale or thickness $\alpha_{\tau}$. The amount of noise is well evaluated everywhere.

the polygon which tends to be detected as noise with the method based on the meaningful thickness (see close up view of image (e) and (f)). Note that the meaningful scale detection appears to be a little more dynamic than the meaningful thickness. From a computational point of view, the meaningful scale method is faster (76 ms and $87 \mathrm{~ms}$ for respectively (b) and (e)) than the meaningful thickness approach $(542 \mathrm{~ms}$ and $485 \mathrm{~ms}$. for respectively (c) and (f) on a Mac OS X 2.8 Ghz Core 2 Duo), but the thickness detection uses an $O\left(n^{2}\right)$ version of the blurred segment detection while all maximal straight segments are computed in linear time according to the number of contour point. More objective time comparisons are let to future works.

Experiment on polygonal curves. The new possibility to detect the meaningful thickness on polygonal curves was experimented in Fig. 8. The first experiment applies the detection on a non uniformly sampled contour (contour of Fig. 5 (a)). The resulting meaningful thickness is everywhere 1 as expected (Fig. 8 (a)). By adding noise on different quadrants of the previous contour, the detector consistently in-

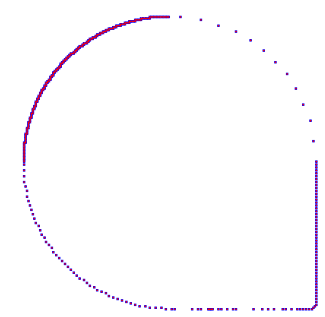

(a)

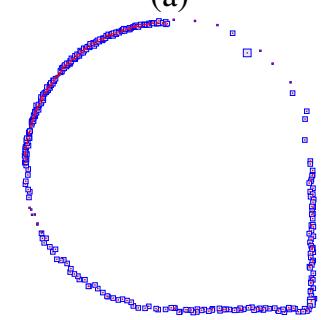

(c)

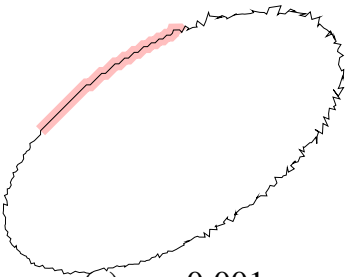

(e) $\varepsilon=0.001$

(Cao, 2003)

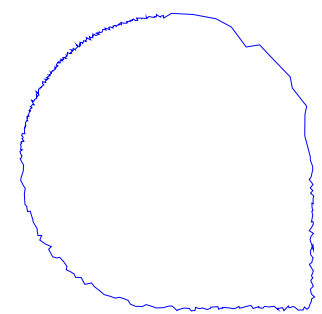

(b)

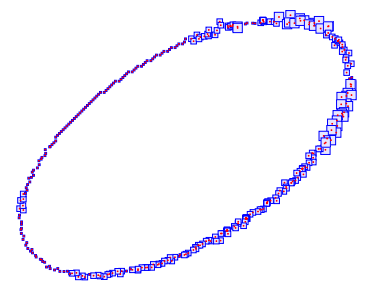

(d)

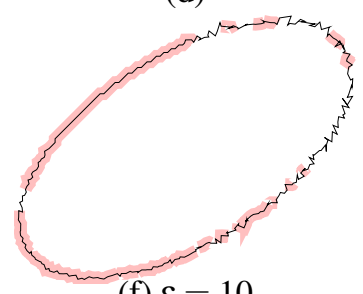

(Cao, 2003)
Figure 8: Meaningful thickness detection on polygonal curves. The polygonal curve (a) was obtained after applying a sampling process defined for each quadrant (the same than for Fig. 5 (a)). The polygon (b) was obtained after adding some noise specifically to each sector and its detected meaningful thickness is represented in (c). (d) shows the same results obtained on the ellipse of the Fig. 5 (a) and $(\mathrm{e}, \mathrm{f})$ show comparisons with the meaningful good continuation method (Cao, 2003) (in thick red plot) obtained with different values of $\varepsilon$.

creases the meaningful thickness (image (c)). The other experiment applied on ellipse also show nice meaningful thickness detection (d).

To apply comparisons with other comparable approach, we have experimented the method of the meaningful good continuation of Cao (Cao, 2003). As briefly described in the introduction and contrary to our method this approach has a parameter $\varepsilon$ which can be tuned to adjust the level of what can be considered as meaningful or not. On results presented on Fig. 8 (e,f) we can see that the meaningful contour parts are well identified and are not in contradiction with the meaningful thickness detection but our approach does not need to set any parameter and can also give directly the meaningful thickness (if it exits).

Application to extract meaningful contours in im- 


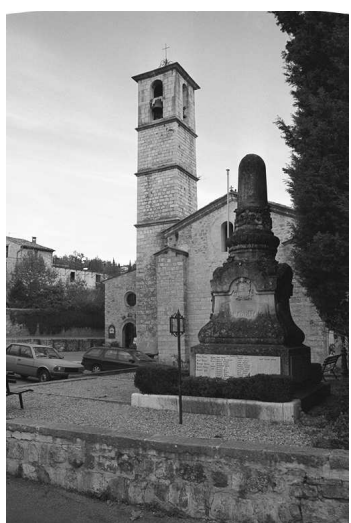

(a) source

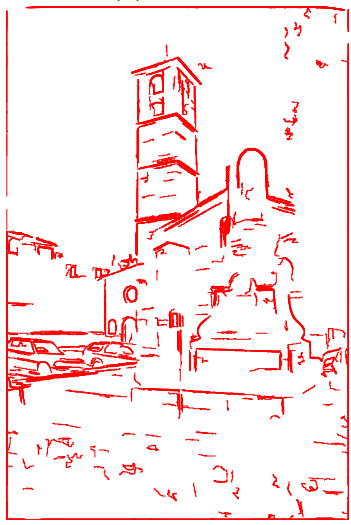

(c) Meaningful contours

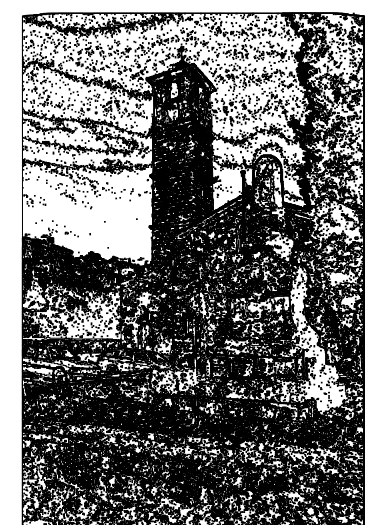

(b) iso contours

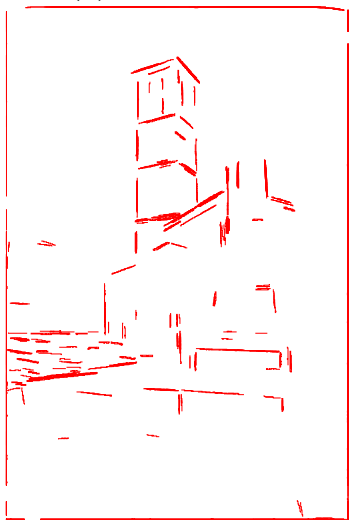

(d) straight parts
Figure 9: Application to meaningful contours extraction (image (c)) using all iso level contours (image (b)). The straight parts obtained from meaningful multi-thickness profile are represented in (d).

ages. The meaningful thickness detection can be applied on every level set of the image. Fig. 9 (b) shows all the set of such a contour extracted after tracking the frontier of the connected components defined from each threshold step. Here 256 gray levels were considered with a step of 10. The image (c) of Fig. 9 show all the contour parts with a meaningful thickness equals to one (i.e. no noise). From all the contours, we also detect the straight contour parts by applying a threshold to the slope of the multi-thickness criterion $\mu_{i_{1}, i_{2}}(P)$ by -0.46 .

\subsection{Simple application for contour smoothing}

This meaningful thickness detection can be used in numerous applications (in particular, in most of the algorithms which use the $\alpha$-thick Blurred Segment primitive). We present here a simple potential application of contour smoothing by taking the meaningful thickness as a constraint for a curve reconstruction. The reconstruction method is an iterative process that computes the new points as a weighted average of its neighbors, constrained by the meaningful thickness (displayed in light blue on Fig. 10 (b,e,h)). Note that these constraints were defined from the meaningful thickness on all non meaningful edges by using linear interpolation between the vertex of the polygon.

The resulting reconstruction visible on images Fig. 10 (c,f,i) show very fine polygonal contours where noise are no more visible. Moreover all initial contour parts with no noise are well preserved after the reconstruction. Another interesting quality is visible with the preservation of all discontinuities in particular for the open contour of Fig. 10 (g-i).

\section{Conclusion}

A new concept of meaningful thickness was presented. The proposed method can be considered as parameter free and can be applied both on discrete or polygonal contour. The results are very promising and open the door to new unsupervised applications. The simple contour smoothing application is a first application which already shows fine results without the need to set any particular parameter. The proposed method is simple to implement and the user can test the algorithm with their own data (Kerautret et al., 2011). The source code is also already available from the ImaGene library (Ima, 2011) and is planned to be integrated as a module in the new DGtal library (DGt, 2011).

\section{REFERENCES}

(2011). DGtal: Digital geometry tools and algorithms library. http://liris.cnrs.fr/dgtal.

(2011). Imagene, Generic digital Image library. http:// gforge.liris.cnrs.frs/projects/imagene.

Bhowmick, P. and Bhattacharya, B. B. (2007). Fast polygonal approximation of digital curves using relaxed straightness properties. IEEE Trans on PAMI, 29(9):1590-1602.

Bruckstein, A. (1991). Self-similarity properties of digitized straight lines. Contemp. Math, 119:1-20.

Cao, F. (2003). Good continuations in digital image level lines. In Proc. of ICCV 2003, volume 1, pages 440 448. IEEE.

Debled-Rennesson, I., Feschet, F., and Rouyer-Degli, J. (2006). Optimal blurred segments decomposition of noisy shapes in linear time. Computers \& Graphics, $30(1)$. 


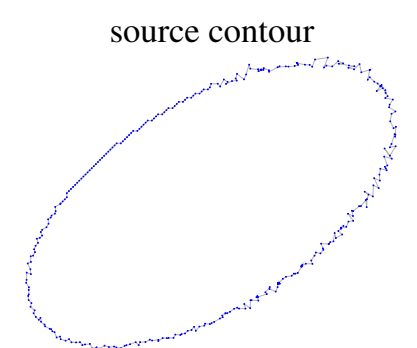

(a)

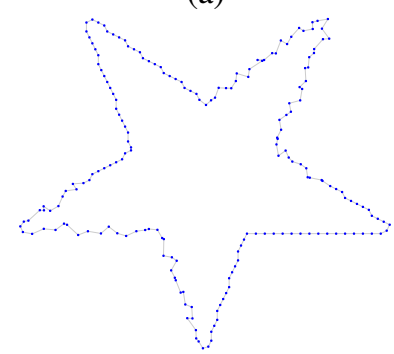

(d)

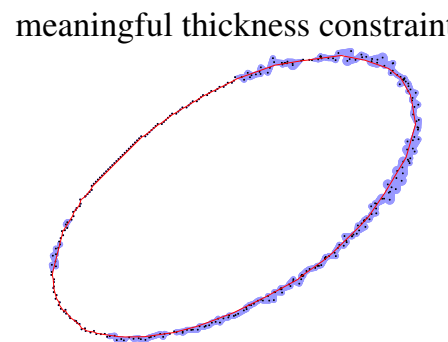

(b)

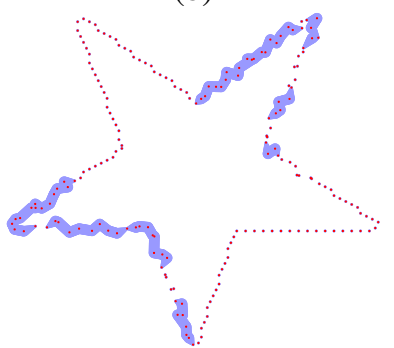

(e) resulting reconstruction

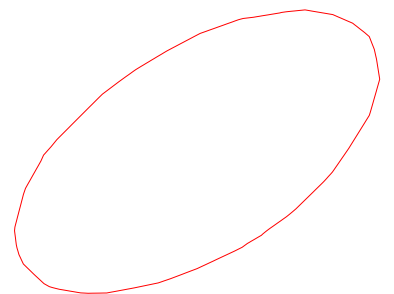

(c)

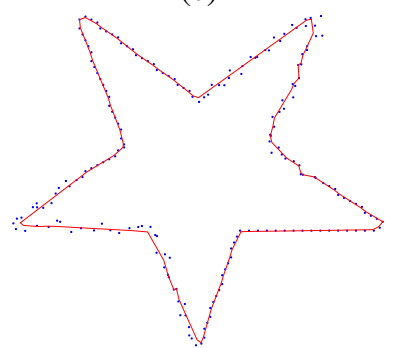

(f)

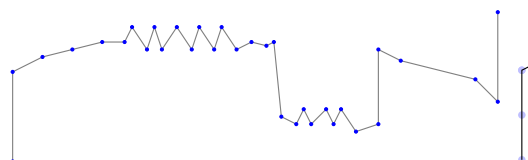

(g)

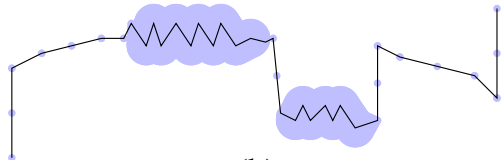

(h)

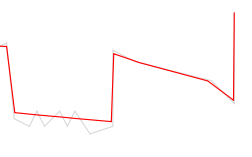

(i)

Figure 10: Application of contour smoothing using the meaningful thickness on several shapes. The initial noisy source contour are given in image $(\mathrm{a}, \mathrm{d}, \mathrm{g})$. The blue areas represent the noise constraints $(\mathrm{b}, \mathrm{e}, \mathrm{h})$ and the resulting smoothed contour is represented in red on $(\mathrm{c}, \mathrm{f}, \mathrm{i})$.

Desolneux, A. (2011). A probabilistic grouping principle to go from pixels to visual structures. In Proc of DGCI, volume 6607 of $L N C S$, pages 1-13. springer.

Desolneux, A., Moisan, L., and Morel, J.-M. (2001). Edge detection by helmholtz principle. Journal of Mathematical Imaging and Vision, 14:271-284.

Dorst, L. and Smeulders, A. (1984). Discrete representation of straight line. IEEE Trans. Patt. Anal. Mach. Intell, 6:450-463.

Faure, A., Buzer, L., and Feschet, F. (2009). Tangential cover for thick digital curves. Pattern Recognition, 42(10):2279 - 2287.

Kerautret, B. and Lachaud, J.-O. (2009a). Curvature estimation along noisy digital contours by approximate global optimization. Pattern Recognition, 42(10): 2265 - 2278.

Kerautret, B. and Lachaud, J.-O. (2009b). Multi-scale analysis of discrete contours for unsupervised noise detection. In Proc. IWCIA, volume 5852 of LNCS, pages 187-200. Springer.

Kerautret, B., Lachaud, J.-O., and Said, M. (2011). Meaningful thickness detection demonstration. http://kerrecherche.iutsd.uhp-nancy.fr/ MeaningfulThickness.

Klette, R. and Rosenfeld, A. (2004). Digital straightness-a review. Discrete Applied Mathematics, 139(1-3):197 -230 .

Lachaud, J.-O. (2006). Espaces non-euclidiens et analyse d'image : modèles déformables riemanniens et discrets, topologie et géométrie discrète. Habilitation à diriger des recherches, Université Bordeaux 1, Talence, France. (en français).

Lachaud, J.-O., Vialard, A., and de Vieilleville, F. (2005). Analysis and comparative evaluation of discrete tangent estimators. In Proc. DGCI, volume 3429 of LNCS, pages 140-251. Springer.

Rosenfeld, A. (1974). Digital straight line segments. IEEE Trans. Comput., 23:1264-1269.

Said, M. (2010). Gomtrie multi-rsolution des objets discrets bruits. PhD thesis, University of Savoies. 